\title{
Objetivos de presión más bajos al estándar no reducirían la morbi-mortalidad en pacientes con hipertensión arterial y enfermedad cardiovascular
}

\author{
Objectives of blood pressure lower than the standard did not reduce morbidity and mortality in patients with \\ hypertension and cardiovascular disease
}

Saiz LC, et al. Cochrane Database of Systematic Reviews 2018, Issue 7. Art. No.: CD010315. PMID: 30027631.

\section{Comentado de:}

Saiz LC, et al. Cochrane Database of Systematic Reviews 2018, Issue 7. Art. No.: CD010315. PMID: $30027631{ }^{1}$

\section{Objetivo}

Determinar si objetivos de presión arterial más bajos $(\leq 135 / 85$ $\mathrm{mmHg}$ ) están asociados a reducción de mortalidad y morbilidad comparado con objetivos de presión arterial estándar" $(\leq 140$ a 160/90 a $100 \mathrm{mmHg}$ ) en pacientes con hipertensión y enfermedad cardiovascular.

\section{Diseño}

Revisión sistemática y meta-análisis.

\section{Fuente de datos y selección de estudios}

La búsqueda se realizó hasta febrero 2018 en el Registro Especializado de Hipertensión Cochrane, Registro Central Cochrane de Ensayos Controlados (CENTRAL), MEDLINE (desde 1946), Embase (desde 1974) y LILACS (desde 1982), junto con la Plataforma de Registro Internacional de Ensayos Clínicos de la Organización Mundial de la Salud y ClinicalTrials.gov. Además se revisaron artículos relevantes sobre trabajos publicados y no publica- dos. No hubo restricciones de idioma.

Se incluyeron ensayos clínicos aleatorizados con al menos 50 pacientes por rama y 6 meses de seguimiento que comparasen objetivos más bajos de tensión arterial $(\leq 135 / 85 \mathrm{mmHg})$ con objetivos estándar ( $\leq 140$ a 160/ 90 a $100 \mathrm{mmHg}$ ).

Se incluyeron adultos con hipertensión y enfermedad cardiovascular establecida (infarto agudo de miocardio, accidente cerebrovascular, enfermedad vascular oclusiva periférica o angina crónica estable).

Dos revisores seleccionaron, evaluaron el riesgo de sesgo y extrajeron los datos de manera independiente.

\section{Medición de resultados principales}

Mortalidad total, efectos adversos graves, eventos cardiovasculares, mortalidad cardiovascular.

\section{Resultados}

Seis estudios cumplieron los criterios de inclusión. El número total de participantes fue 9.484 (5.301 asignados al objetivo más bajo y 4.183 al objetivo estándar). La media de seguimiento fue 3,7 años.

Los resultados principales se resumen en la tabla 1.

Tabla 1. Resultados principales de objetivos de presión arterial más bajos en comparación con los objetivos de presión arterial estándar. Notas: ${ }^{a}$ ECA - Ensayo clínico aleatorizado; IC: intervalo de confianza.

\begin{tabular}{|c|c|c|c|}
\hline Resultado & $\begin{array}{l}\text { Riesgo Relativo } \\
\text { (IC } 95 \% \text { ) }\end{array}$ & $\begin{array}{l}\text { Nro. participantes } \\
\text { (estudios) }\end{array}$ & $\begin{array}{l}\text { Calidad de evidencia } \\
\text { (GRADE) }\end{array}$ \\
\hline Mortalidad total & $1,06(0,91$ a 1,23$)$ & $\begin{array}{l}9.484 \\
(6 \mathrm{ECA})^{a}\end{array}$ & $\begin{array}{l}\oplus \oplus \oplus \ominus \\
\text { Moderada }\end{array}$ \\
\hline Efectos adversos graves & $1,01(0,94$ a 1,08$)$ & $\begin{array}{l}9.484 \\
(6 \mathrm{ECA})\end{array}$ & $\begin{array}{l}\oplus \oplus \ominus \ominus \\
\text { Baja }\end{array}$ \\
\hline Eventos cardiovasculares & $0,89(0,80$ a 1,00$)$ & $\begin{array}{l}9.484 \\
(6 \mathrm{ECA})\end{array}$ & $\begin{array}{l}\oplus \oplus \ominus \ominus \\
\text { Baja }\end{array}$ \\
\hline Mortalidad cardiovascular & $1,03(0,82$ a 1,29$)$ & $\begin{array}{l}9.484 \\
(6 \mathrm{ECA})\end{array}$ & $\begin{array}{l}\oplus \oplus \oplus \ominus \\
\text { Moderada }\end{array}$ \\
\hline Retiro por efectos adversos & $8,16(2,06$ a 32,28$)$ & $\begin{array}{l}690 \\
(2 \mathrm{ECA})\end{array}$ & $\begin{array}{l}\oplus \ominus \ominus \ominus \\
\text { Muy baja }\end{array}$ \\
\hline
\end{tabular}

\section{Conclusiones}

En la actualidad, la evidencia es insuficiente para justificar objetivos de presión arterial más baja $(\leq 135 / 85 \mathrm{mmHg})$ en personas con hipertensión y enfermedad cardiovascular establecida, con evidencia muy limitada sobre efectos adversos, lo que lleva a una alta incertidumbre.

\section{Fuentes de financiamiento}

Sistema de salud de Navarra, España. European Social Fund Operational Programme. Universidad de British Columbia, Vancouver, Canadá. 


\section{Comentario}

Este trabajo se puede leer en el contexto de la guía de diagnóstico y manejo de hipertensión arterial, que publicara en 2017 la Asociación Americana del Corazón y el Colegio Americano de Cardiología ${ }^{2}$.

En dicha guía se proponían objetivos de tensión arterial de $130 / 80 \mathrm{mmHg}$ para pacientes con enfermedad cardiovascular establecida o riesgo elevado (mayor al $10 \%$ de eventos a 10 años) de padecerla. También se definía 130/80 mmHg como punto de corte para que una persona sea considerada hipertensa.

El trabajo de Saiz et al ${ }^{1}$, una revisión sistemática de buena calidad metodológica, aporta evidencia en sentido contrario. Si bien se observó una tendencia a la reducción de eventos cardiovasculares con objetivos más bajos, esta no fue estadísticamente significativa.

Esto sugiere que al perseguir objetivos más bajos de tensión arterial, exponemos a los pacientes a mayores costos y a un riesgo aumentado de eventos adversos sin un beneficio neto de salud.

Por otro lado, incluso con los objetivos actuales, existe en el mundo y en la Argentina en particular un gran número de personas hipertensas no diagnosticadas o que no reciben tratamiento adecuado por distintos motivos ${ }^{3}$ y considero que los recursos de salud pública deberían concentrarse prioritariamente en esta población.

\section{Conclusiones del comentador}

Los objetivos estándar de tensión arterial de $<140 / 90 \mathrm{mmHg}$ son apropiados para la mayoría de los pacientes en prevención secundaria.

Sebastián Sguiglia [ Servicio de Medicina Familiar y Comunitaria, Hospital Italiano de Buenos Aires. sebastian.sguiglia@hospitalitaliano.org.ar ]

Sguiglia S Objetivos de presión más bajos al estándar no reducirían la morbi-mortalidad en pacientes con hipertensión arterial y enfermedad cardiovascular. Evid Actual Pract Ambul. 2019;4():e001101. Comentado de: Saiz LC, y col. Blood pressure targets for the treatment of people with hypertension and cardiovascular disease. Cochrane Database of Systematic Reviews 2018, Issue 7. Art. No.: CD010315. PMID: 30027631.

\section{Referencias}

1. Saiz LC, Gorricho J, Garjón J, Celaya MC, Erviti J, Leache L. Blood pressure targets for the treatment of people with hypertension and cardiovascular disease. Cochrane Database Syst Rev. 2018;7(7).

2. Whelton PK, Carey RM, Aronow WS. PCNA Guideline for the Prevention, Detection, Evaluation, and Management of High Blood Pressure in Adults: Executive Summary: A Report of the American College of Cardiology/American Heart Association Task Force on Clinical Practice Guidelines. Hypertension. 2017;71(6):1269-1324. published correction appears in Hypertension. published correction appears in Hypertension.

3. Delucchi AM, Majul CR, Vicario A, Cerezo G, Fábregues G. Registro Nacional de Hipertensión Arterial. Características epidemiológicas de la hipertensión arterial en Argentina. Estudio RENATA 2. Rev Fed Arg Cardiol. 2017;46:91-95. 